\title{
Stress-Induced Hyperglycemia, but Not Diabetic Hyperglycemia, Is Associated with Higher Mortality in Patients with Isolated Moderate and Severe Traumatic Brain Injury: Analysis of a Propensity Score-Matched Population
}

\author{
Cheng-Shyuan Rau ${ }^{1,+}$, Shao-Chun $\mathrm{Wu}^{2,+}$, Yi-Chun Chen ${ }^{3}$, Peng-Chen Chien ${ }^{3}$, \\ Hsiao-Yun Hsieh ${ }^{3}$, Pao-Jen Kuo ${ }^{3}$ and Ching-Hua Hsieh ${ }^{3, *}$ (iD \\ 1 Department of Neurosurgery, Kaohsiung Chang Gung Memorial Hospital, Chang Gung University College \\ of Medicine, Kaohsiung City 833, Taiwan; ersh2127@cloud.cgmh.org.tw \\ 2 Department of Anesthesiology, Kaohsiung Chang Gung Memorial Hospital, \\ Chang Gung University College of Medicine, Kaohsiung City 833, Taiwan; shaochunwu@gmail.com \\ 3 Department of Plastic Surgery, Kaohsiung Chang Gung Memorial Hospital, Chang Gung University College \\ of Medicine, Kaohsiung City 833, Taiwan; libe320@yahoo.com.tw (Y.-C.C.); \\ VENU_CHIEN@hotmail.com (P.-C.C.); sylvia19870714@hotmail.com (H.-Y.H.); \\ bow110470@gmail.com (P.-J.K.) \\ * Correspondence: m93chinghua@gmail.com; Tel.: +886-7-7327476 \\ + These authors contributed equally to this work.
}

Received: 21 September 2017; Accepted: 1 November 2017; Published: 3 November 2017

\begin{abstract}
Background: Admission hyperglycemia is associated with higher morbidity and mortality in patients with traumatic brain injury (TBI). Stress-induced hyperglycemia (SIH), a form of hyperglycemia induced by the stress response, is associated with increased patient mortality following TBI. However, admission hyperglycemia occurs not only in SIH but also in patients with diabetic hyperglycemia (DH). Current information regarding whether trauma patients with SIH represent a distinct group with differential outcomes compared to those with $\mathrm{DH}$ remains limited. Methods: Serum glucose concentration $\geq 200 \mathrm{mg} / \mathrm{dL}$ upon arrival at the emergency department was defined as hyperglycemia. Presence of diabetes mellitus (DM) was determined by patient history and/or admission glycated hemoglobin ( $\mathrm{HbA} 1 \mathrm{c}$ ) level $\geq 6.5 \%$. In the present study, the patient cohort included those with moderate and severe TBI, as defined by an Abbreviated Injury Scale (AIS) score $\geq 3$ points in the head, and excluded those who had additional AIS scores $\geq 3$ points in any other region of the body. A total of 1798 adult patients with isolated moderate to severe TBI were allocated into four groups: SIH $(n=140)$, DH $(n=187)$, diabetic normoglycemia (DN, $n=186)$, and non-diabetic normoglycemia (NDN, $n=1285$ ). Detailed patient information was retrieved from the Trauma Registry System at a level I trauma center between 1 January 2009, and 31 December 2015. Unpaired Student's $t$ - and Mann-Whitney $U$-tests were used to analyze normally and non-normally distributed continuous data, respectively. Categorical data were compared using the Pearson chi-square or two-sided Fisher's exact tests. Matched patient populations were allocated in a 1:1 ratio according to propensity scores calculated by NCSS software. Logistic regression was used to evaluate the effect of SIH and DH on the adjusted mortality outcome. Results: In patients with isolated moderate to severe TBI, the presence of SIH and DH led to 9.1-fold and 2.3-fold higher odds of mortality, respectively, than patients with NDN. After adjusting for confounding factors, including sex and age, pre-existing co-morbidities, existence of different kinds of intracerebral hemorrhage, and injury severity, patients with SIH still had 6.6-fold higher odds of mortality than those with NDN; however, DH did not present significantly higher adjusted mortality odds. SIH and DH presented different effects on outcomes after TBI. The results also suggested that the pathophysiological effect associated with SIH was different from that of DH. Conclusions: This study demonstrated that patients
\end{abstract}


with SIH and DH had significantly higher mortality than patients with NDN. However, the adjusted mortality was significantly higher only in the selected propensity score-matched patients with SIH and not in those with DH.

Keywords: traumatic brain injury (TBI); stress-induced hyperglycemia (SIH); diabetic hyperglycemia (DH); diabetes mellitus (DM); mortality

\section{Introduction}

Admission hyperglycemia is associated with higher morbidity and mortality in patients with traumatic brain injury (TBI) [1-3]. Patients with severe TBI have significantly higher serum glucose levels than those with mild TBI [1]. A stress response associated with TBI can induce a form of stress-induced hyperglycemia (SIH) [4-6], which also commonly occurs in patients with critical illnesses such as burn injuries [7], myocardial infarction [8], stroke [9,10], and trauma [11-15]. The hyperglycemia following TBI is suspected to contribute to tissue lactic acidosis in the brain and result in neuronal injury [16-18]. An association between SIH and increased mortality following TBI has been reported [5,19]. Many studies have also consistently shown SIH to be associated with higher morbidity and mortality rates in trauma patients $[9,14,15,20]$.

However, admission hyperglycemia may occur not only as SIH but also as diabetic hyperglycemia (DH). Few studies have evaluated the differential effects of SIH and DH on the outcomes in the trauma population. Trauma patients with SIH have a $>2$-fold increase in mortality risk (relative risk (RR) 2.41, 95\% confidence interval (CI) 1.81-3.23) than those without $\mathrm{SIH}$, whereas patients with DH have a nonsignificant, near-50\% increase in mortality risk (RR 1.47, 95\% CI 0.92-2.36) [21]. Current information regarding whether patients with TBI having SIH represent a distinct group with different outcomes than those with DH remains limited. Bosarge et al. reported that patients with severe TBI and SIH had a 50\% greater mortality (95\% CI 1.13-1.95) than TBI patients with nondiabetic normoglycemia (NDN) after adjusting for age, sex, injury mechanism, Injury Severity Score (ISS), Revised Trauma Score (RTS), and lactic acid level [5]. On the other hand, DH patients did not have a significantly higher mortality [5]. However, this study did not exclude patients with polytrauma and did not compare the patients by adjusting for pre-existing co-morbidities and the existence of different kinds of intracerebral hemorrhage, which may impact the assessment of the mortality outcome. Therefore, the present study aimed to perform a further assessment of the effect of SIH and $\mathrm{DH}$ on the outcomes of the presence of hyperglycemia in patients with isolated moderate to severe TBI. We excluded patients with polytrauma and selected a propensity score-matched patient cohort to reduce the effect of differences in sex, age, pre-existing co-morbidities, and the existence of different kinds of intracerebral hemorrhage, such as epidural hemorrhage (EDH), subdural hemorrhage (SDH), subarachnoidal hemorrhage (SAH), and intracerebral hemorrhage (ICH), and injury severity in the patient population on the outcome assessment. The primary hypothesis of the present study was that patients with SIH had a similar or worse outcome than those with $\mathrm{DH}$, based on the mortality rate as the primary measured outcome.

\section{Methods}

\subsection{Study Population}

This study was approved by the Institutional Review Board (IRB) of the Kaohsiung Chang Gung Memorial Hospital, a Level I regional trauma center in southern Taiwan [22,23] before its implementation (reference number 201701210B0). According to IRB regulations, the requirement for informed consent was waived. The present retrospective study reviewed the data of all adult hospitalized trauma patients ( $\geq 20$ years old) registered in the Trauma Registry System of the hospital 
from 1 January 2009 to 31 December 2015. In the present study, the patient cohort included those with moderate and severe TBI, defined by an Abbreviated Injury Scale (AIS) score $\geq 3$ points in the head (AIS 3-4 and 5 indicate moderate and severe TBI, respectively) [24]. To avoid the confounding effect of injuries to other body regions, polytrauma patients [25] with additional AIS scores $\geq 3$ points in any other region of the body were excluded from the study; thus, the included patients were defined as having isolated moderate and severe TBI. In addition, only those patients with available data on serum glucose level in the emergency department as well as those patients with a history of diabetes mellitus $(\mathrm{DM})$ or glycated hemoglobin $(\mathrm{HbA} 1 \mathrm{c})$ level data were included, whereas patients with incomplete data were excluded from the study. A serum glucose concentration $\geq 200 \mathrm{mg} / \mathrm{dL}$ upon arrival at the emergency department was defined as hyperglycemia. DM was diagnosed by patient history and/or admission $\mathrm{HbA} 1 \mathrm{c}$ level $\geq 6.5 \%$, according to the current recommendations for DM diagnosis from the American Diabetes Association [26]. SIH was diagnosed by serum glucose concentration $\geq 200 \mathrm{mg} / \mathrm{dL}$ in patients without DM and DH was diagnosed by serum glucose concentration $\geq 200 \mathrm{mg} / \mathrm{dL}$ in patients with DM. Diabetic normoglycemia (DN) and NDN was determined by serum glucose concentration $<200 \mathrm{mg} / \mathrm{dL}$ in patients with and without DM, respectively. In the present study, the enrolled patients were allocated into four exclusive groups based on above definitions (Figure 1). The retrieved patient information for the study included the following: sex; age; existence of EDH, $\mathrm{SDH}, \mathrm{SAH}$, and $\mathrm{ICH}$; co-morbidities such as hypertension (HTN), coronary artery disease (CAD), congestive heart failure (CHF), cerebral vascular accident (CVA), and end-stage renal disease (ESRD); serum glucose level at the emergency department; HbA1c level; Glasgow coma scale (GCS) scale; ISS, which was expressed as the median and interquartile range (IQR, Q1-Q3); hospital length of stay (LOS); rates of admission into the intensive care unit (ICU); and in-hospital mortality.

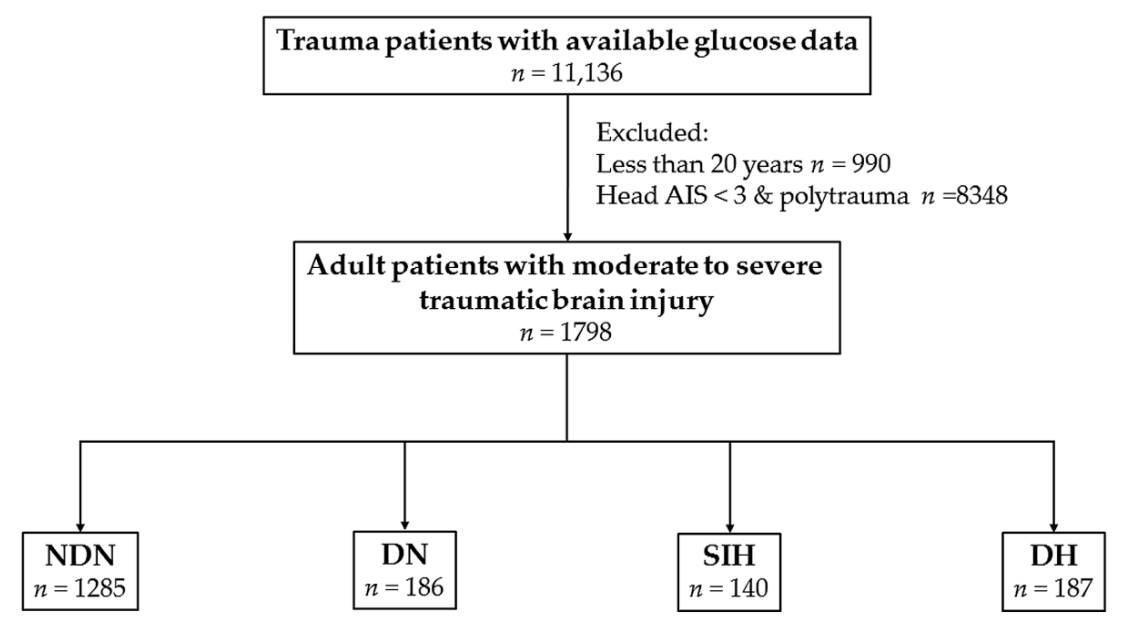

Figure 1. Flowchart of the allocation of patients with isolated moderate and severe traumatic brain injuries into four exclusive groups of non-diabetic normoglycemia (NDN), diabetic normoglycemia (DN), stress-induced hyperglycemia (SIH), and diabetic hyperglycemia (DH).

\subsection{Statistical Analysis}

We performed the statistical analyses using IBM SPSS Statistics for Windows, version 20.0 (IBM Corp., Armonk, NY, USA) and NCSS 10 software (NCSS Statistical Software, Kaysville, UT, USA). The primary outcome of the study was in-hospital mortality. Two-sided Fisher's exact or Pearson chi-square tests were used to compare categorical data. Odds ratios (ORs) with 95\% CIof the associated conditions of the patients were presented. The normally distributed continuous and non-normally distributed data were analyzed with unpaired Student's $t$ - and Mann-Whitney $U$-tests, respectively, and presented as mean \pm standard deviation. To minimize the confounding effects of the baseline characteristics of the compared patient populations due to a non-randomized assignment, a 1:1 propensity score-matched study group was created using the Greedy method with a 0.2 caliper width 
using NCSS 10 software to assess the effect of SIH and DH on the outcomes. The propensity scores were calculated using a logistic regression model with the following covariates: sex, age, co-morbidities, types of intracranial hemorrhage, and ISS. After adjusting for these confounding factors, cox regression was used to evaluate the effects of SIH and DH on the primary and secondary outcomes against those of NDN. $p$-values $<0.05$ were defined as statistically significant.

\section{Results}

\subsection{Characteristics and Outcomes of Patients with SIH}

There were 1798 adult patients with isolated moderate to severe TBI in the present study (Figure 1 ). These patients were allocated into four groups: $\mathrm{SIH}(n=140), \mathrm{DH}(n=187), \mathrm{DN}(n=186)$, and NDN $(n=1285)$. As shown in Table 1, no significant differences in sex and age were observed between the $\mathrm{SIH}$ and NDN groups. The SIH group had significantly higher rates of $\mathrm{EDH}, \mathrm{SDH}$, and $\mathrm{ICH}$, but there was no difference between the rate of SAH in the SIH and NDN groups. No significant difference in the rates of pre-existing co-morbidities was observed between the SIH and NDN groups. On the other hand, more patients had a GCS $\leq 8$ and fewer had a GCS score $\geq 13$, the SIH group had a significantly lower GCS score than the NDN group ( $7.7 \pm 4.7$ vs. $12.4 \pm 3.8$, respectively; $p<0.001)$. The SIH group had a significantly higher ISS (median (IQR: Q1-Q3), 20 (16-25]) than the NDN group (16 (13-20]). In addition, more patients with SIH had an ISS $\geq 25$, but fewer patients with SIH had an ISS $<16$ than those with NDN. Regarding the patient outcomes, the SIH group had a higher proportion of patients admitted to the ICU $(83.6 \%$ vs. $66.4 \%$, respectively; $p<0.001)$ and 9.1 -fold higher odds of mortality than the NDN group $(95 \%$ CI $6.10-13.48 ; p<0.001)$. There was no difference in the hospital LOS between the SIH and NDN groups.

Table 1. Comparison of the characteristics and outcomes of the SIH and NDN groups of patients.

\begin{tabular}{|c|c|c|c|c|}
\hline Variables & $\begin{array}{c}\text { SIH } \\
n=140\end{array}$ & $\begin{array}{c}\text { NDN } \\
n=1285\end{array}$ & $\begin{array}{l}\text { Odds Ratio } \\
\text { (95\% CI) }\end{array}$ & $p$ \\
\hline Sex & & & & 0.984 \\
\hline Female, $n(\%)$ & $50(35.7)$ & $460(35.8)$ & $1.0(0.69-1.43)$ & \\
\hline Male, $n(\%)$ & $90(64.3)$ & $825(64.2)$ & $1.0(0.70-1.44)$ & \\
\hline Age (years) & $52.4 \pm 18.7$ & $54.3 \pm 19.8$ & - & 0.297 \\
\hline \multicolumn{5}{|l|}{ Hemorrhage type } \\
\hline $\mathrm{EDH}, n(\%)$ & $45(32.1)$ & $273(21.2)$ & $1.8(1.20-2.57)$ & 0.003 \\
\hline $\mathrm{SDH}, n(\%)$ & $90(64.3)$ & $688(53.5)$ & $1.6(1.09-2.25)$ & 0.015 \\
\hline $\mathrm{SAH}, n(\%)$ & $76(54.3)$ & $634(49.3)$ & $1.2(0.86-1.73)$ & 0.266 \\
\hline $\mathrm{ICH}, n(\%)$ & $30(21.4)$ & $137(10.7)$ & $2.3(1.47-3.55)$ & $<0.001$ \\
\hline \multicolumn{5}{|l|}{ Co-morbidity } \\
\hline $\mathrm{HTN}, n(\%)$ & $36(25.7)$ & $326(25.4)$ & $1.0(0.68-1.52)$ & 0.929 \\
\hline $\mathrm{CAD}, n(\%)$ & $4(2.9)$ & $42(3.3)$ & $0.9(0.31-2.47)$ & 1.000 \\
\hline $\mathrm{CHF}, n(\%)$ & $2(1.4)$ & $8(0.6)$ & $2.3(0.49-11.00)$ & 0.257 \\
\hline CVA, $n(\%)$ & $2(1.4)$ & $55(4.3)$ & $0.3(0.08-1.34)$ & 0.102 \\
\hline ESRD, $n(\%)$ & $0(0.0)$ & $2(0.2)$ & - & 1.000 \\
\hline GCS & $7.7 \pm 4.7$ & $12.4 \pm 3.8$ & - & $<0.001$ \\
\hline$\leq 8$ & $89(63.6)$ & $255(19.8)$ & $7.0(4.87-10.21)$ & $<0.001$ \\
\hline $9-12$ & $18(12.9)$ & 157 (12.2) & $1.1(0.63-1.79)$ & 0.827 \\
\hline$\geq 13$ & $33(23.6)$ & $873(67.9)$ & $0.1(0.10-0.22)$ & $<0.001$ \\
\hline ISS, median (IQR) & $20(16-25)$ & $16(13-20)$ & - & $<0.001$ \\
\hline$<16$ & $23(16.4)$ & $466(36.3)$ & $0.3(0.22-0.55)$ & $<0.001$ \\
\hline $16-24$ & $65(46.4)$ & $678(52.8)$ & $0.8(0.55-1.10)$ & 0.154 \\
\hline$\geq 25$ & $52(37.1)$ & $141(11.0)$ & $4.8(3.26-7.05)$ & $<0.001$ \\
\hline Hospital LOS (days) & $14.4 \pm 21.1$ & $12.2 \pm 12.5$ & - & 0.236 \\
\hline ICU admission, $n(\%)$ & 117 (83.6) & $853(66.4)$ & $2.6(1.62-4.09)$ & $<0.001$ \\
\hline Mortality, $n(\%)$ & $58(41.4)$ & $93(7.2)$ & $9.1(6.10-13.48)$ & $<0.001$ \\
\hline
\end{tabular}

$\mathrm{CAD}=$ coronary artery disease; $\mathrm{CHF}=$ congestive heart failure; $\mathrm{CI}=$ confidence interval; $\mathrm{CVA}=$ cerebral vascular accident; $\mathrm{EDH}$ = epidural hemorrhage; ESRD = end-stage renal disease; GCS = Glasgow coma scale; $\mathrm{HTN}$ = hypertension; ICH = intracerebral hemorrhage; ICU = intensive care unit; $\mathrm{IQR}=$ interquartile range; ISS = injury severity score; LOS = length of stay; NDN = nondiabetic normoglycemia; $\mathrm{SAH}$ = subarachnoid hemorrhage; $\mathrm{SDH}$ = subdural hemorrhage; $\mathrm{SIH}$ = stress-induced hyperglycemia. 


\subsection{Characteristics and Outcomes of Patients with DH}

There was a significantly higher predominance of women and senior patients in the DH group than in the NDN group (Table 2). The DH group had a significantly higher rate of EDH than the NDN group, but no differences in the rates of $\mathrm{SDH}, \mathrm{SAH}$, and $\mathrm{ICH}$ were observed between the $\mathrm{DH}$ and NDN groups. Higher odds of HTN and CAD, but not other co-morbidities, were observed in the $\mathrm{DH}$ group than in the NDN group. There was no significant difference in GCS scores between the DH and NDN groups (12.1 \pm 4.2 vs. $12.4 \pm 3.8$, respectively; $p<0.001)$, regardless of patient GCS score classifications of $\leq 8$, between 9 and 12 , and $\geq 13$. There was no significant difference between the ISS (median (IQR: Q1-Q3), 16 (14-20) in the DH group and that in the NDN group (16 (13-20)). In addition, fewer DH patients had an ISS $<16$ than in the NDN group. There was no difference in the hospital LOS and proportion of patients admitted to the ICU between the DH and NDN groups. However, patients in the DH group had 2.2-fold higher odds of mortality than those in the NDN group (95\% CI 1.37-3.42; $p=0.001)$.

Table 2. Comparison of the characteristics and outcomes of the DH and NDN groups of patients.

\begin{tabular}{ccccc}
\hline Variables & $\begin{array}{c}\text { DH } \\
n=\mathbf{1 8 7}\end{array}$ & $\begin{array}{c}\text { NDN } \\
\boldsymbol{n}=\mathbf{1 2 8 5}\end{array}$ & $\begin{array}{c}\text { Odds Ratio } \\
\mathbf{( 9 5 \% ~ C I )}\end{array}$ & $p$ \\
\hline Sex & & & & $<0.001$ \\
Female, $n(\%)$ & $92(49.2)$ & $460(35.8)$ & $1.7(1.28-2.37)$ & \\
Male, $n(\%)$ & $95(50.8)$ & $825(64.2)$ & $0.6(0.42-0.78)$ & \\
Age (years) & $65.6 \pm 12.0$ & $54.3 \pm 19.8$ & - & $<0.001$ \\
\hline Hemorrhage type & & & & \\
EDH, $n(\%)$ & $25(13.4)$ & $273(21.2)$ & $0.6(0.37-0.89)$ & 0.012 \\
SDH, $n(\%)$ & $104(55.6)$ & $688(53.5)$ & $1.1(0.80-1.48)$ & 0.595 \\
SAH, $n(\%)$ & $78(41.7)$ & $634(49.3)$ & $0.7(0.54-1.00)$ & 0.051 \\
ICH, $n(\%)$ & $22(11.8)$ & $137(10.7)$ & $1.1(0.69-1.80)$ & 0.650 \\
\hline Co-morbidity & & & & \\
HTN, $n(\%)$ & $116(62.0)$ & $326(25.4)$ & $4.8(3.49-6.63)$ & $<0.001$ \\
CAD, $n(\%)$ & $20(10.7)$ & $42(3.3)$ & $3.5(2.03-6.18)$ & $<0.001$ \\
CHF, $n(\%)$ & $4(2.1)$ & $8(0.6)$ & $3.5(1.04-11.70)$ & 0.055 \\
CVA, $n(\%)$ & $14(7.5)$ & $55(4.3)$ & $1.8(0.99-3.32)$ & 0.053 \\
ESRD, $n(\%)$ & $0(0.0)$ & $2(0.2)$ & - & 1.000 \\
\hline GCS $\quad 12.1 \pm 4.2$ & $12.4 \pm 3.8$ & - & 0.320 \\
$\leq 8$ & $44(23.5)$ & $255(19.8)$ & $1.2(0.86-1.79)$ & 0.242 \\
$9-12$ & $19(10.2)$ & $157(12.2)$ & $0.8(0.49-1.34)$ & 0.418 \\
$\geq 13$ & $124(66.3)$ & $873(67.9)$ & $0.9(0.67-1.29)$ & 0.656 \\
\hline ISS, median (IQR) & $16(14-20)$ & $16(13-20)$ & - & 0.183 \\
$<16$ & $50(26.7)$ & $466(36.3)$ & $0.6(0.46-0.90)$ & 0.011 \\
$16-24$ & $108(57.8)$ & $678(52.8)$ & $1.2(0.90-1.67)$ & 0.201 \\
$\geq 25$ & $29(15.5)$ & $141(11.0)$ & $1.5(0.97-2.30)$ & 0.070 \\
\hline Hospital LOS (days) & $13.3 \pm 12.0$ & $12.2 \pm 12.5$ & - & 0.296 \\
ICU admission, $n(\%)$ & $134(71.7)$ & $853(66.4)$ & $1.3(0.91-1.80)$ & 0.151 \\
Mortality, $n(\%)$ & $27(14.4)$ & $93(7.2)$ & $2.2(1.37-3.42)$ & 0.001 \\
\hline
\end{tabular}

$\mathrm{CAD}=$ coronary artery disease; $\mathrm{CHF}=$ congestive heart failure; $\mathrm{CI}=$ confidence interval; $\mathrm{CVA}=$ cerebral vascular accident; $\mathrm{DH}$ = diabetic hyperglycemia; $\mathrm{EDH}$ = epidural hemorrhage; $\mathrm{ESRD}$ = end-stage renal disease; GCS = Glasgow coma scale; HTN = hypertension; $\mathrm{ICH}=$ intracerebral hemorrhage; $\mathrm{ICU}=$ intensive care unit; $\mathrm{IQR}$ = interquartile range; ISS = injury severity score; LOS = length of stay; NDN = nondiabetic normoglycemia; $\mathrm{SAH}=$ subarachnoid hemorrhage; $\mathrm{SDH}=$ subdural hemorrhage.

\subsection{Adjusted Outcomes of Patients with SIH and with DH}

Propensity score-matched patients were selected to reduce the effect of differences in sex, age, pre-existing co-morbidities, types of intracranial hemorrhage, and injury severity of the patient population on the outcome assessment. As shown in Table 3, in selected 122 well-balanced pairs of SIH and NDN patients, there were no significant differences in sex, age, co-morbidity, types of intracranial hemorrhage, and ISS between the two patient cohorts. The logistic regression analysis of these pairs of patients showed that those with SIH had 6.6-fold higher odds of mortality (95\% CI 2.58-16.91; $p<0.001$ ) than those with NDN (test for coefficients mortality: $\mathrm{b}=1.887$; Wald $=15.462 ; p<0.001$, 
test for model coefficients: -2 Log Likelihood $\left.=146.041, \lambda^{2}(1)=20.632, p<0.001\right)$. In addition, in the 163 well-balanced pairs of patients with DH and NDN (Table 4), there were also no significant differences in sex, age, co-morbidity, types of intracranial hemorrhage, and ISS between the two patient cohorts. The logistic regression analysis of these pairs of patients did not show a significant difference of odds of mortality (OR 1.4; 95\% CI 0.68-2.71; $p=0.386$ ) between DH and NDN (test for coefficients mortality: $\mathrm{b}=0.305$; Wald $=0.752 ; p=0.386$, test for model coefficients: -2 Log Likelihood $=225.205$, $\left.\lambda^{2}(1)=0.758, p=0.384\right)$.

Table 3. Comparison of the mortality outcomes in the selected propensity-score matched cohort of SIH and NDN groups of patients.

\begin{tabular}{|c|c|c|c|c|c|}
\hline \multirow[b]{2}{*}{ Variables } & \multicolumn{5}{|c|}{ Propensity Score-Matched Cohort } \\
\hline & $\begin{array}{c}\text { SIH } \\
n=122\end{array}$ & $\begin{array}{c}\text { NDN } \\
n=122\end{array}$ & $\begin{array}{c}\text { Odds Ratio } \\
\text { (95\% CI) }\end{array}$ & $p$ & Standardized Difference \\
\hline Sex & & & & 1.000 & \\
\hline Female, $n(\%)$ & $40(32.8)$ & $40(32.8)$ & $1.0(0.59-1.71)$ & & $0.00 \%$ \\
\hline Male, $n(\%)$ & $82(67.2)$ & $82(67.2)$ & $1.0(0.59-1.71)$ & & $0.00 \%$ \\
\hline Age (years) & $50.9 \pm 18.7$ & $51.0 \pm 17.9$ & - & 0.992 & $-0.13 \%$ \\
\hline \multicolumn{6}{|l|}{ Co-morbidity } \\
\hline HTN, $n(\%)$ & $25(20.5)$ & $25(20.5)$ & $1.0(0.54-1.86)$ & 1.000 & $0.00 \%$ \\
\hline $\mathrm{CAD}, n(\%)$ & $1(0.8)$ & $1(0.8)$ & $1.0(0.06-16.17)$ & 1.000 & $0.00 \%$ \\
\hline $\mathrm{CHF}, n(\%)$ & $0(0.0)$ & $0(0.0)$ & - & - & - \\
\hline CVA, $n(\%)$ & $1(0.8)$ & $1(0.8)$ & $1.0(0.06-16.17)$ & 1.000 & $0.00 \%$ \\
\hline ESRD, $n(\%)$ & $0(0.0)$ & $0(0.0)$ & - & - & - \\
\hline $\mathrm{EDH}, n(\%)$ & $42(34.4)$ & $42(34.4)$ & $1.0(0.59-1.70)$ & 1.000 & $0.00 \%$ \\
\hline $\mathrm{SDH}, n(\%)$ & $83(68.0)$ & $83(68.0)$ & $1.0(0.58-1.71)$ & 1.000 & $0.00 \%$ \\
\hline $\mathrm{SAH}, n(\%)$ & 63 (51.6) & 63 (51.6) & $1.0(0.61-1.65)$ & 1.000 & $0.00 \%$ \\
\hline $\mathrm{ICH}, n(\%)$ & $21(17.2)$ & $21(17.2)$ & $1.0(0.51-1.94)$ & 1.000 & $0.00 \%$ \\
\hline ISS, median (IQR) & $17(16-25)$ & $17(16-25)$ & - & 0.898 & $0.00 \%^{+}$ \\
\hline Mortality, $n(\%)$ & $49(40.2)$ & $21(17.2)$ & $6.6(2.58-16.91)$ & $<0.001$ & \\
\hline
\end{tabular}

$\mathrm{CAD}=$ coronary artery disease; $\mathrm{CHF}=$ congestive heart failure; $\mathrm{CI}=$ confidence interval; $\mathrm{CVA}=$ cerebral vascular accident; $\mathrm{EDH}=$ epidural hemorrhage; $\mathrm{ESRD}=$ end-stage renal disease; HTN = hypertension; $\mathrm{ICH}=$ intracerebral hemorrhage; IQR = interquartile range; ISS = injury severity score; NDN = nondiabetic normoglycemia; $\mathrm{SAH}=$ subarachnoid hemorrhage; $\mathrm{SDH}=$ subdural hemorrhage; $\mathrm{SIH}=$ stress-induced hyperglycemia. ${ }^{+}$indicates difference of interquartile range.

Table 4. Comparison of mortality outcomes in the selected propensity-score matched cohort of DH and NDN groups of patients.

\begin{tabular}{|c|c|c|c|c|c|}
\hline \multirow[b]{2}{*}{ Variables } & \multicolumn{5}{|c|}{ Propensity Score-Matched Cohort } \\
\hline & $\begin{array}{c}\text { DH } \\
n=163\end{array}$ & $\begin{array}{c}\text { NDN } \\
n=163\end{array}$ & $\begin{array}{l}\text { Odds Ratio } \\
(95 \% \text { CI })\end{array}$ & $p$ & Standardized Difference \\
\hline Sex & & & & 1.000 & \\
\hline Female, $n(\%)$ & $74(45.4)$ & $74(45.4)$ & $1.0(0.65-1.55)$ & & $0.00 \%$ \\
\hline Male, $n(\%)$ & 89 (54.6) & 89 (54.6) & $1.0(0.65-1.55)$ & & $0.00 \%$ \\
\hline Age (years) & $65.0 \pm 12.3$ & $65.3 \pm 13.1$ & - & 0.852 & $-2.07 \%$ \\
\hline \multicolumn{6}{|l|}{ Co-morbidity } \\
\hline HTN, $n(\%)$ & 97 (59.5) & 97 (59.5) & $1.0(0.64-1.56)$ & 1.000 & $0.00 \%$ \\
\hline CAD, $n(\%)$ & $6(3.7)$ & $6(3.7)$ & $1.0(0.32-3.17)$ & 1.000 & $0.00 \%$ \\
\hline CHF, $n(\%)$ & $1(0.6)$ & $1(0.6)$ & $1.0(0.06-16.13)$ & 1.000 & $0.00 \%$ \\
\hline $\mathrm{CVA}, n(\%)$ & $11(6.7)$ & $11(6.7)$ & $1.0(0.42-2.38)$ & 1.000 & $0.00 \%$ \\
\hline ESRD, $n(\%)$ & $0(0.0)$ & $0(0.0)$ & - & - & - \\
\hline $\mathrm{EDH}, n(\%)$ & $21(12.9)$ & $21(12.9)$ & $1.0(0.52-1.91)$ & 1.000 & $0.00 \%$ \\
\hline $\mathrm{SDH}, n(\%)$ & 87 (53.4) & 87 (53.4) & $1.0(0.65-1.55)$ & 1.000 & $0.00 \%$ \\
\hline $\mathrm{SAH}, n(\%)$ & $69(42.3)$ & $69(42.3)$ & $1.0(0.64-1.55)$ & 1.000 & $0.00 \%$ \\
\hline $\mathrm{ICH}, n(\%)$ & $15(9.2)$ & $15(9.2)$ & $1.0(0.47-2.12)$ & 1.000 & $0.00 \%$ \\
\hline ISS, median (IQR) & $16(13-20)$ & $16(13-20)$ & - & 0.916 & $0.00 \%^{\dagger}$ \\
\hline Mortality, $n(\%)$ & $23(14.1)$ & $18(11.0)$ & $1.4(0.68-2.71)$ & 0.386 & \\
\hline
\end{tabular}

$\mathrm{CAD}=$ = coronary artery disease; $\mathrm{CHF}=$ congestive heart failure; $\mathrm{CI}=$ confidence interval; $\mathrm{CVA}=$ cerebral vascular accident; $\mathrm{DH}$ = diabetic hyperglycemia; $\mathrm{EDH}$ = epidural hemorrhage; ESRD = end-stage renal disease; $\mathrm{HTN}=$ hypertension; ICH = intracerebral hemorrhage; IQR = interquartile range; ISS = injury severity score; $\mathrm{NDN}=$ nondiabetic normoglycemia; $\mathrm{SAH}=$ subarachnoid hemorrhage; $\mathrm{SDH}=$ subdural hemorrhage. ${ }^{\dagger}$ indicates difference of interquartile range. 


\section{Discussion}

In the present study of patients with isolated moderate to severe TBI, those with SIH and DH had 9.1-fold and 2.3-fold higher odds of mortality, respectively, than those with NDN. After adjusting for confounding factors, including sex and age, pre-existing co-morbidities, the existence of different kinds of intracerebral hemorrhage, and injury severity, patients with SIH still had 6.6-fold higher odds of mortality than those with NDN; however, the same was not observed in patients with DH, who did not present significantly higher mortality odds. This result was in accordance with the report by Bosarge et al. in which SIH in patients with severe TBI was associated with higher mortality than patients with NDN after adjusting for age, sex, injury mechanism, ISS, RTS, and lactic acid level; this increased mortality was not observed in those patients with DH [5]. Notably, in the present study, the adjusted 6.6-fold odds of mortality of SIH was much higher than that reported by Bosarge et al. who reported a 50\% increased adjusted mortality in SIH patients with severe TBI [5]. We believe that the discrepancy may be partly attributed to the exclusion of patients with polytrauma from the present study. This discrepancy may also be partly attributed to the evaluation method with a propensity-score matched population by adjusting for pre-existing co-morbidities and different kinds of intracerebral hemorrhage. In the present study, the SIH group had a significantly higher ISS than the NDN and even the DH groups. The occurrences of a variety of intracerebral hemorrhages differed between the SIH and NDN groups and even between the SIH and DH groups. In addition, the patients with less severe injuries in the present study (moderate to severe TBI) than the population studied by Bosarge et al. (severe TBI) may also have contributed to the discrepancy, as the effect of hyperglycemia is less easy to measure in patients with high mortality. The above adjustments are expected to reduce bias in the assessment of mortality outcomes and infer a different extent of the odds of mortality. An adjustment to reduce bias in comparison is important during the assessment; for example, our previous study which explored the morbidities associated with SIH for all trauma patients had revealed that even the SIH had a 2.9-fold higher odds of pneumonia (95\% CI 1.68-4.93; $p<0.001)$ and a 4.8 -fold higher odds of acute renal failure ( $95 \%$ CI 2.15-10.52; $p<0.001$ ) than the NDN, there were no differences in the rates of pneumonia and acute renal failure between SIH and NDN after adjustment by propensity-score matching [27].

Notably, hyperglycemia following TBI is associated with injury severity and poor outcomes [28,29]. Patients with severe TBI have significantly higher serum glucose levels than those with mild TBI [1]. The causes of SIH is secondary to a state of increased hepatic output of glucose, diminished insulin production, and insulin resistance in peripheral tissue in the presence of excessive adrenal cortical output (glucagon, growth hormone, catecholamine, and glucocorticoid) as well as high circulating levels of cytokines like tumor necrosis factor- $\alpha$, interleukin-1, and interleukin-6 [20,30]. Up to 10 times of these greater adrenal cortical outputs causing SIH was found [31]. In contrast, DH is a chronic process associated with microvascular changes due to prolonged hyperglycemia [20]. In the present study, $\mathrm{SIH}$ and DH differed in their effect on the outcomes of patients after TBI. Although the mechanism for the detrimental effects of these two hyperglycemia states is unknown, the results indicate that the pathophysiological effect associated with SIH might be different from that of DH. In this study, the SIH could be provided as a marker for worse mortality. However, whether SIH is directly harmful to the subject remained debatable. Historically, SIH appears to be a marker of disease severity and the hyperglycemia and insulin resistance induced in the setting of acute illness had been thought to be a protective mechanism that increases the host's chances of survival [31]. In experimental animal models, the results are inconsistent to determine whether SIH is harmful, neutral, or beneficial for outcomes following TBI [32,33]. Furthermore, currently no definitive statements can be made as to whether aggressive treatment of hyperglycemia actually benefits outcomes of trauma patients [34-36].

The present study had several limitations. The first limitation is represented by the inherent selection bias associated with the retrospective study design. The cause of mortality of these patients with TBI was unknown in this retrospective study, and thus may result in a selection bias in the comparison of outcomes between groups. Second, patients declared dead at the scene of the accident 
or upon hospital arrival were not included in the database, which may have led to a selection bias, considering that the mortality rate was the primary outcome. Third, as patients with DH may have some degree of stress response invoking their hyperglycemia, $\mathrm{SIH}$ and $\mathrm{DH}$ are not mutually exclusive. Currently, the SIH in diabetes has not been explored and lacks a well-established definition. In addition, because we did not measure the levels of stress response hormones or catecholamine, it was not possible for us to specifically identify whether stress might be more responsible for the hyperglycemia in the patients with DH. Furthermore, the use of glycemic control is not performed under strict guidelines at our hospital and may vary among patients with TBI. In addition, some diabetes drugs targeting the glucagon-like peptide-1 receptor, such as metformin and thiazolidinediones, may promote neuronal survival by affecting brain metabolism, neuroinflammation, and regeneration [37]. However, their effect on mortality could not be corrected in this retrospective study, and thus may result in a bias in the outcome assessment. Although the studies on the use and effect of glycemic control have been inconclusive, this may have resulted in a bias in the outcome assessment. This also indicates that defining the appropriate treatment for SIH remains an area of ongoing investigation.

\section{Conclusions}

The results of this study demonstrated worse outcomes for patients with stress-induced hyperglycemia (SIH), but not diabetic hyperglycemia ( $\mathrm{DH})$, among patients with isolated moderate to severe traumatic brain injury (TBI) with respect to mortality, after controlling for age, sex, pre-existing co-morbidities, the existence of different kinds of intracerebral hemorrhage, and Injury Severity Score (ISS).

Acknowledgments: We appreciated the Biostatistics Center, Kaohsiung Chang Gung Memorial Hospital for statistics work. This research was supported by a grant from CMRPG8F0261 for Ching-Hua Hsieh.

Author Contributions: Cheng-Shyuan Rau wrote the manuscript, Shao-Chun Wu was involved in the literature review, Yi-Chun Chen and Peng-Chen Chien performed the statistical analyses, Hsiao-Yun Hsieh proofread the manuscript, Pao-Jen Kuo was responsible for the integrity of registered data, and Ching-Hua Hsieh designed the study and contributed to the data analysis and interpretation. All authors read and approved the final manuscript.

Conflicts of Interest: The authors declare that they have no competing interests.

\section{References}

1. Alexiou, G.A.; Lianos, G.; Fotakopoulos, G.; Michos, E.; Pachatouridis, D.; Voulgaris, S. Admission glucose and coagulopathy occurrence in patients with traumatic brain injury. Brain Inj. 2014, 28, 438-441. [CrossRef] [PubMed]

2. Prisco, L.; Iscra, F.; Ganau, M.; Berlot, G. Early predictive factors on mortality in head injured patients: A retrospective analysis of 112 traumatic brain injured patients. J. Neurosurg. Sci. 2012, 56, 131-136. [PubMed]

3. Salim, A.; Hadjizacharia, P.; Dubose, J.; Brown, C.; Inaba, K.; Chan, L.S.; Margulies, D. Persistent hyperglycemia in severe traumatic brain injury: An independent predictor of outcome. Am. Surg. 2009, 75, 25-29. [PubMed]

4. Khajavikhan, J.; Vasigh, A.; Kokhazade, T.; Khani, A. Association between Hyperglycaemia with Neurological Outcomes Following Severe Head Trauma. J. Clin. Diagn. Res. JCDR 2016, 10. [CrossRef] [PubMed]

5. Bosarge, P.L.; Shoultz, T.H.; Griffin, R.L.; Kerby, J.D. Stress-induced hyperglycemia is associated with higher mortality in severe traumatic brain injury. J. Trauma Acute Care Surg. 2015, 79, 289-294. [CrossRef] [PubMed]

6. Kinoshita, K. Traumatic brain injury: Pathophysiology for neurocritical care. J. Intensiv. Care 2016, 4, 29. [CrossRef] [PubMed]

7. Gauglitz, G.G.; Herndon, D.N.; Jeschke, M.G. Insulin resistance postburn: Underlying mechanisms and current therapeutic strategies. J. Burn Care Res. Off. Publ. Am. Burn Assoc. 2008, 29, 683-694. [CrossRef] [PubMed]

8. Smit, J.W.; Romijn, J.A. Acute insulin resistance in myocardial ischemia: Causes and consequences. Semin. Cardiothorac. Vasc. Anesth. 2006, 10, 215-219. [CrossRef] [PubMed]

9. Ray, B.; Ludwig, A.; Yearout, L.K.; Thompson, D.M.; Bohnstedt, B.N. Stress-Induced Hyperglycemia after Spontaneous Subarachnoid Hemorrhage and Its Role in Predicting Cerebrospinal Fluid Diversion. World Neurosurg. 2017, 100, 208-215. [CrossRef] [PubMed] 
10. Yang, C.J.; Liao, W.I.; Wang, J.C.; Tsai, C.L.; Lee, J.T.; Peng, G.S.; Lee, C.H.; Hsu, C.W.; Tsai, S.H. Usefulness of glycated hemoglobin A1c-based adjusted glycemic variables in diabetic patients presenting with acute ischemic stroke. Am. J. Emerg. Med. 2017, 35, 1240-1246. [CrossRef] [PubMed]

11. Sung, J.; Bochicchio, G.V.; Joshi, M.; Bochicchio, K.; Tracy, K.; Scalea, T.M. Admission hyperglycemia is predictive of outcome in critically ill trauma patients. J. Trauma 2005, 59, 80-83. [CrossRef] [PubMed]

12. Richards, J.E.; Kauffmann, R.M.; Zuckerman, S.L.; Obremskey, W.T.; May, A.K. Relationship of hyperglycemia and surgical-site infection in orthopaedic surgery. J. Bone Jt. Surg. Am. 2012, 94, 1181-1186. [CrossRef] [PubMed]

13. Richards, J.E.; Kauffmann, R.M.; Obremskey, W.T.; May, A.K. Stress-induced hyperglycemia as a risk factor for surgical-site infection in nondiabetic orthopedic trauma patients admitted to the intensive care unit. J. Orthop. Trauma 2013, 27, 16-21. [CrossRef] [PubMed]

14. Mraovic, B.; Suh, D.; Jacovides, C.; Parvizi, J. Perioperative hyperglycemia and postoperative infection after lower limb arthroplasty. J. Diabetes Sci. Technol. 2011, 5, 412-418. [CrossRef] [PubMed]

15. Leto, R.; Desruelles, D.; Gillet, J.B.; Sabbe, M.B. Admission hyperglycaemia is associated with higher mortality in patients with hip fracture. Eur. J. Emerg. Med. 2015, 22, 99-102. [CrossRef] [PubMed]

16. Oddo, M.; Schmidt, J.M.; Mayer, S.A.; Chiolero, R.L. Glucose control after severe brain injury. Curr. Opin. Clin. Nutr. Metab. Care 2008, 11, 134-139. [CrossRef] [PubMed]

17. Zygun, D.A.; Steiner, L.A.; Johnston, A.J.; Hutchinson, P.J.; Al-Rawi, P.G.; Chatfield, D.; Kirkpatrick, P.J.; Menon, D.K.; Gupta, A.K. Hyperglycemia and brain tissue $\mathrm{pH}$ after traumatic brain injury. Neurosurgery 2004, 55, 877-881, discussion 882. [CrossRef] [PubMed]

18. Rostami, E.; Bellander, B.M. Monitoring of glucose in brain, adipose tissue, and peripheral blood in patients with traumatic brain injury: A microdialysis study. J. Diabetes Sci. Technol. 2011, 5, 596-604. [CrossRef] [PubMed]

19. Bonizzoli, M.; Zagli, G.; Lazzeri, C.; Degl'Innocenti, S.; Gensini, G.; Peris, A. Early insulin resistance in severe trauma without head injury as outcome predictor? A prospective, monocentric pilot study. Scand. J. Trauma Resusc. Emerg. Med. 2012, 20, 69. [CrossRef] [PubMed]

20. Bosarge, P.L.; Kerby, J.D. Stress-induced hyperglycemia: Is it harmful following trauma? Adv. Surg. 2013, 47, 287-297. [CrossRef] [PubMed]

21. Kerby, J.D.; Griffin, R.L.; MacLennan, P.; Rue, L.W., 3rd. Stress-induced hyperglycemia, not diabetic hyperglycemia, is associated with higher mortality in trauma. Ann. Surg. 2012, 256, 446-452. [CrossRef] [PubMed]

22. Hsieh, C.H.; Hsu, S.Y.; Hsieh, H.Y.; Chen, Y.C. Differences between the sexes in motorcycle-related injuries and fatalities at a Taiwanese level I trauma center. Biomed. J. 2017, 40, 113-120. [CrossRef] [PubMed]

23. Hsieh, C.H.; Liu, H.T.; Hsu, S.Y.; Hsieh, H.Y.; Chen, Y.C. Motorcycle-related hospitalizations of the elderly. Biomed. J. 2017, 40, 121-128. [CrossRef] [PubMed]

24. Savitsky, B.; Givon, A.; Rozenfeld, M.; Radomislensky, I.; Peleg, K. Traumatic brain injury: It is all about definition. Brain Inj. 2016, 30, 1194-1200. [CrossRef] [PubMed]

25. Butcher, N.; Balogh, Z.J. AIS > 2 in at least two body regions: A potential new anatomical definition of polytrauma. Injury 2012, 43, 196-199. [CrossRef] [PubMed]

26. American Diabetes Association. Diagnosis and classification of diabetes mellitus. Diabetes Care 2012, 35, S64-S71.

27. Rau, C.S.; Wu, S.C.; Chen, Y.C.; Chien, P.C.; Hsieh, H.Y.; Kuo, P.J.; Hsieh, C.H. Higher Mortality in Trauma Patients Is Associated with Stress-Induced Hyperglycemia, but Not Diabetic Hyperglycemia: A Cross-Sectional Analysis Based on a Propensity-Score Matching Approach. Int. J. Environ. Res. Public Health 2017, 14, 1161. [CrossRef] [PubMed]

28. Rovlias, A.; Kotsou, S. The influence of hyperglycemia on neurological outcome in patients with severe head injury. Neurosurgery 2000, 46, 335-342, discussion 342-343. [CrossRef] [PubMed]

29. Young, B.; Ott, L.; Dempsey, R.; Haack, D.; Tibbs, P. Relationship between admission hyperglycemia and neurologic outcome of severely brain-injured patients. Ann. Surg. 1989, 210, 466-473. [CrossRef] [PubMed]

30. McCowen, K.C.; Malhotra, A.; Bistrian, B.R. Stress-induced hyperglycemia. Crit. Care Clin. 2001, 17, 107-124. [CrossRef]

31. Marik, P.E.; Bellomo, R. Stress hyperglycemia: An essential survival response! Crit. Care Med. 2013, 41, e93-e94. [CrossRef] [PubMed] 
32. Cherian, L.; Hannay, H.J.; Vagner, G.; Goodman, J.C.; Contant, C.F.; Robertson, C.S. Hyperglycemia increases neurological damage and behavioral deficits from post-traumatic secondary ischemic insults. J. Neurotrauma 1998, 15, 307-321. [CrossRef] [PubMed]

33. Hill, J.; Zhao, J.; Dash, P.K. High blood glucose does not adversely affect outcome in moderately brain-injured rodents. J. Neurotrauma 2010, 27, 1439-1448. [CrossRef] [PubMed]

34. Butcavage, K. Glycemic control and intensive insulin protocols for neurologically injured patients. J. Neurosci. Nurs. 2012, 44, E1-E9. [CrossRef] [PubMed]

35. Kramer, A.H.; Roberts, D.J.; Zygun, D.A. Optimal glycemic control in neurocritical care patients: A systematic review and meta-analysis. Crit. Care (Lond. Engl.) 2012, 16, R203. [CrossRef] [PubMed]

36. Scalea, T.M.; Bochicchio, G.V.; Bochicchio, K.M.; Johnson, S.B.; Joshi, M.; Pyle, A. Tight glycemic control in critically injured trauma patients. Ann. Surg. 2007, 246, 605-612. [CrossRef] [PubMed]

37. Patrone, C.; Eriksson, O.; Lindholm, D. Diabetes drugs and neurological disorders: New views and therapeutic possibilities. Lancet Diabetes Endocrinol. 2014, 2, 256-262. [CrossRef]

(C) 2017 by the authors. Licensee MDPI, Basel, Switzerland. This article is an open access article distributed under the terms and conditions of the Creative Commons Attribution (CC BY) license (http:/ / creativecommons.org/licenses/by/4.0/). 